\title{
Caracterización clínica del síndrome nefrótico en infantes de Neiva
}

\section{Clinical characterisation of nephrotic syndrome in children in Neiva}

\author{
Diana C León', Ana M Agudelo', Jorge Ramos², Milton D Ibarra ${ }^{3}$
}

\begin{abstract}
Resumen
El Síndrome Nefrótico es la enfermedad glomerular crónica más prevalente en pediatría, en los Estados Unidos presenta una incidencia anual de 2,0 a 2,7 casos por 100.000 niños, y una prevalencia acumulada de 16 por 100.000 (1). El departamento del Huila, carece de información actualizada que permita establecer y unificar objetivos claros en el diagnóstico y tratamiento de esta patología en la población pediátrica. Materiales y Métodos: Se realizó un estudio descriptivo tipo corte transversal de las características clínicas y epidemiológicas del Síndrome Nefrótico en niños atendidos en el Hospital Universitario de Neiva, entre marzo de 2011 y diciembre de 2013; la información se recolectó mediante revisión de historias clínicas. Se incluyeron 67 pacientes con diagnóstico confirmado. Resultados: la edad promedio de debut fue 46 meses (0-175 meses), en su mayoría de género masculino (53,7\%). El 19\% presentaron corticoresistencia, la patología más frecuente fue la glomeruloesclerosis focal y segmentaria (GEFS) (60\%). La presencia de hipertensión arterial al debut o durante el seguimiento, hematuria, hipocomplementemia y dislipidemia persistente se asoció con cortico-resistencia $(P<0,01)$. Se observó cambios en las medidas antropométricas de talla y peso en los pacientes al inicio y final del seguimiento $(P<0,01)$. Conclusión: El Síndrome Nefrótico en esta región es una patología prevalente, con presencia de cortico-resistencia y progresión a enfermedad renal crónica y muerte, por lo tanto es imperativo su diagnóstico temprano, tratamiento y seguimiento oportuno para evitar complicaciones a futuro.
\end{abstract}

Palabras clave: Síndrome Nefrótico; Proteinuria, Hiperlipidemia

\begin{abstract}
Nephrotic Syndrome is a chronic glomerular disease more commonly found in children. The United States has an annual incidence of 2.0 to 2.7 cases per 100,000 children and an accumulated prevalence of 16 per 100,000 . The department of Huila, Colombia lacks up-to-date information that would allow the establishment and standardisation of clear objectives in the diagnosis and treatment of this disease in the paediatric population.

Equipment and Methods: A descriptive, cross-sectional study was carried out of the clinical and epidemiological characteristics of Nephrotic Syndrome in children treated at Neiva's Hospital Universitario between March 2011 and December 2013. The information was collected by reviewing clinical records. 67 patients with a confirmed diagnosis were included.

Results: The average age of onset was 46 months (0-175 months) and patients were mainly male (53.7\%). 19\% showed cortical resistance, the most common disease was focal segmental glomerulosclerosis (FSGS) (60\%). High blood pressure at the beginning, or during follow-up, haematuria, hypocomplementemia
\end{abstract}

1 MD. Pediatra. Universidad Surcolombiana de Neiva.

2 Enf. Epidemiólogo, MSc Epidemiología Clínica, Doctorando en Salud Pública. Universidad Surcolombiana de Neiva.

3 MD. Pediatra especialista en Nefrología. Docente Especialidad médica de Pediatría. Universidad Surcolombiana deNeiva. Correspondencia: Jorge Ramos. Correo electrónico: jormos2806@gmail.com

Recibido: 29/07/2014 Revisado: 13/12/2014 Aceptado: 30/05/2015 
and persistent dyslipidaemia are associated with cortical resistance $(P<0.01)$. Changes in the anthropometric measurements of height and weight were analysed in the patients at the beginning and at the end of the follow-up ( $P<0.01$ ).

Conclusion: Nephrotic Syndrome is a common disease in the region of Neiva and the presence of cortical resistance and the progression to chronic kidney disease and death is also seen. Therefore, early diagnosis, treatment and timely follow-up is imperative in order to avoid complications in the future.

Key words: Nephrotic Syndrome; Proteinuria; Hyperlipidaemia

\section{Introducción}

El Síndrome Nefrótico (SN) está caracterizado por la presencia de proteinuria masiva ( $>40 \mathrm{mg} / \mathrm{m} 2 / \mathrm{h}$ ), hipoalbuminemia $(2,5 \mathrm{~g} / \mathrm{dl})$, edemas e hiperlipidemia (colesterol $>200 \mathrm{mg} / \mathrm{dl})^{(1)}$; es una de las patologías renales más frecuentes en pediatría, con una incidencia de 2 casos por 100.000 habitantes $^{(2)}$. De acuerdo con el tipo histopatológico y con algunas características poblacionales, la respuesta al tratamiento y la progresión a enfermedad renal crónica tienen una amplia variabilidad. La enfermedad de cambios mínimos es la de mayor frecuencia de presentación, la de mejor respuesta al tratamiento inmunosupresor y la de menor afección de la función renal, en contraste con la Glomeruloesclerosis focal segmentaria (GEFS) que en un porcentaje importante $(60 \%)$ es resistente al tratamiento y evoluciona hacia enfermedad renal crónica ${ }^{(2)}$. La edad de inicio, el grupo étnico y el sexo, entre otras, son características importantes que orientan hacia los diferentes tipos histológicos de la enfermedad. El diagnóstico temprano, la evaluación de factores de riesgo asociados a la presentación clínica y la respuesta al tratamiento permiten determinar el seguimiento y el pronóstico renal de estos pacientes. Aquí radica la importancia de conocerlos para así poder elaborar guías de manejo adaptadas a las características sociodemográficas e histopatológicas en la enfermedad en nuestra población infantil.

A pesar de la relevancia, en la región no se cuenta con estudios actualizados sobre $\mathrm{SN}$ en la población pediátrica $\mathrm{y}$ se desconoce las características sociodemográficas y clínicas de los niños y niñas que padecen de síndrome nefrótico en el departamento del Huila.

Se realizó esta investigación para identificar las características clínicas de los pacientes con $\mathrm{SN}$ atendidos en dos instituciones de la ciudad de Neiva desde marzo del 2011 a diciembre del 2013.

\section{Materiales y métodos}

Se realizó un estudio descriptivo de tipo transversal, en pacientes con Síndrome Nefrótico atendidos entre marzo de 2011 hasta diciembre de 2013, en el servicio de nefrología pediátrica del Hospital Universitario de Neiva (HUN); la información se recolectó mediante la técnica de revisión de historias clínicas.

La población objeto fueron 67 historias clínicas, y corresponden a los pacientes menores de 18 años con diagnóstico de SN confirmado por clínica e histopatológicamente.
Para el diagnóstico, se tuvo en cuenta la presencia de proteinuria masiva: $>40 \mathrm{mg} / \mathrm{m} 2 / \mathrm{h}$; hipoalbuminemia: $2,5 \mathrm{~g} / \mathrm{dl}$; edemas e hiperlipidemia: colesterol $>200 \mathrm{mg} / \mathrm{dl}^{(2)}$

Se elaboró un formulario según las variables a estudiar, que permitiera obtener la información necesaria que fue diligenciado directamente por los investigadores.

La validez del instrumento la realizó un experto en nefrología pediátrica y en epidemiologia. La prueba piloto se realizó aplicando el formulario a 10 historias clínicas escogidas al azar, con el fin de valorar la confiabilidad de este y para detectar posibles sesgos de medición y poder corregirlos.

Para el procesamiento de la información se utilizó una codificación establecida por el grupo investigador y la tabulación se realizó mediante tablas y gráficos estadísticos utilizando el programa SPSS Statistics 14, de IBM, New York, USA.

Se realizó análisis univariado realizando la descripción por proporciones en las variables categóricas, y se hizo uso de las medidas de tendencia central (promedio o mediana) y de dispersión (desviación estándar y rango) para las variables numéricas; previamente se verifico el comportamiento normal de ellas mediante la prueba estadística Shapiro-Wilk. En las variables de interés se realizó un análisis bivariado para determinar posibles asociaciones utilizando la prueba estadística chi cuadrado con confiabilidad del 95\%; cuando en la tabla de $2 \times 2$ existía valores esperados menores de 5, se realizó la prueba exacta de Fisher. Se aplicaron las siguientes pruebas estadísticas, rangos de Wilcoxon para determinar diferencias de mediana y correlación de Spearman para comprobar relación entre una variable numérica y categórica. Finalmente, se utilizó el gráfico de Kaplan Meier para determinar supervivencia en los grupos de cortico resistencia y cortico sensible (Figura 1). Todas las pruebas se hicieron con una confiabilidad del $95 \%$.

\section{Consideraciones Éticas}

De acuerdo con el Articulo 11, del Título II de la resolución $n^{\circ} 8430$ del 4 de octubre de 1993, esta investigación se clasifica como Investigación sin riesgo, siendo además aprobada por el Comité de Investigación, Extensión y Docencia Institucional.

\section{Resultados}

El presente estudio incluye el análisis de 67 casos de SN en menores de 18 años de edad durante el periodo de marzo 2011 a diciembre 2013 . 


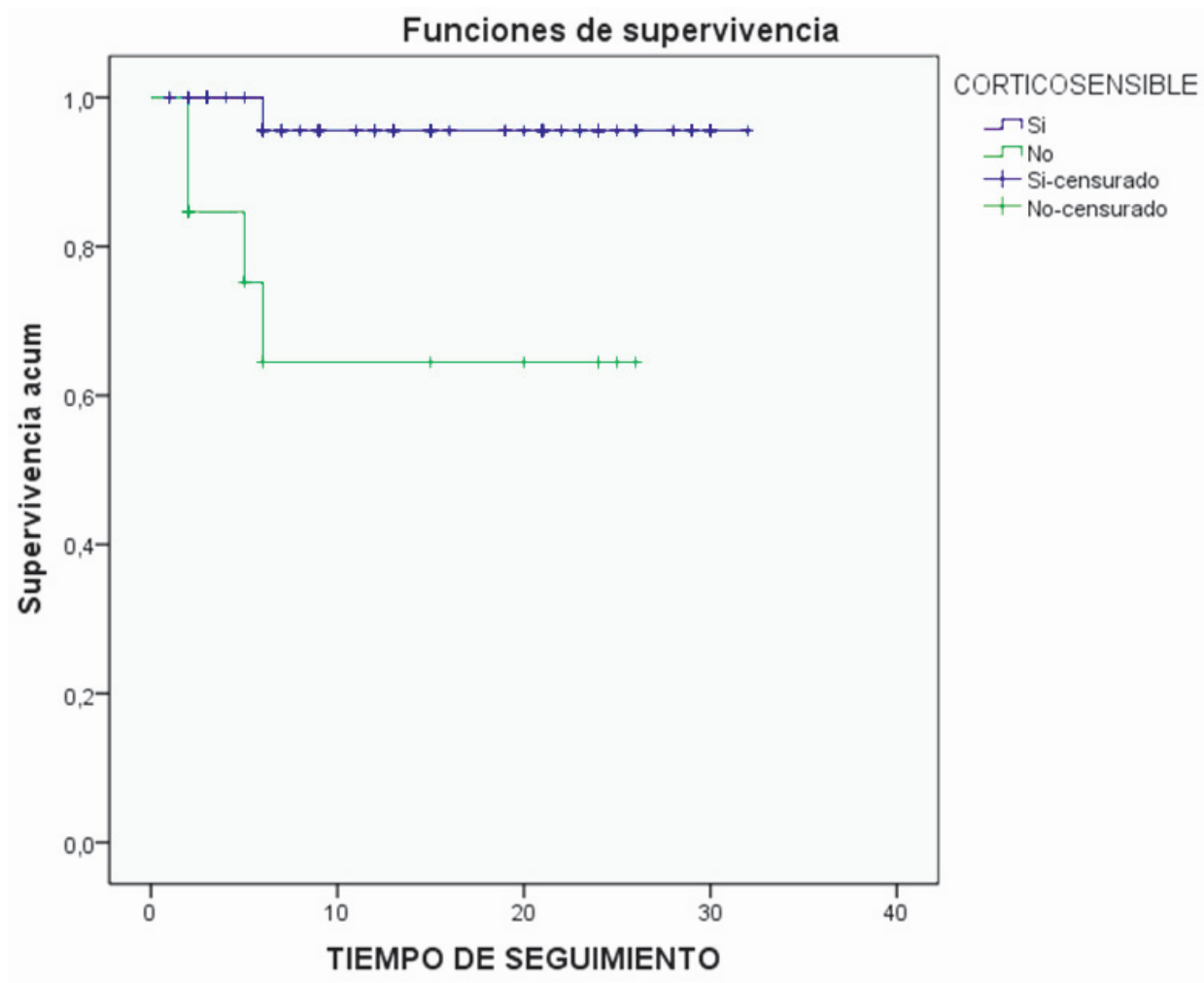

Figura 1. Supervivencia en Cortico - Sensible y Cortico - Resistente.

Los pacientes con SN debutan a la edad de casi cuatros años (46,25 meses), el $89,5 \%$ debutan entre los 13 y 120 meses de edad y su primera visita con especialista es a los 7 años (Tabla 1).

Con relación a los cambios antropométricos de los pacientes al inicio y final del control, se observa que la relación talla/edad continuo muy similar, siendo en su mayoría normal para la edad (80,6\%); el $48 \%$ de los niños menores de 5 años tenían exceso de peso (sobrepeso u obesidad) al inicio y al final disminuyo al $42,9 \%$, debido a que ya se encontraban siendo mayores de 5 años.

En mayores de 5 años el $40,42 \%$ tenían exceso de peso (sobrepeso u obesidad) al inicio, y finalizaron 34,63\% aumentando la proporción de los obesos, respecto a los de sobrepeso (Tabla 2). Se obtuvo una ganancia promedio de peso y talla de 3 kilos y $6 \mathrm{Cm}$ en un tiempo promedio de seguimiento de 14,31 meses.

Del total de pacientes con debut de SN todos presentaron edema, hipoalbuminemia, hipercolesterolemia y proteinuria severa como criterios de diagnóstico. El 3\% presentó complemento bajo y $11,9 \%$ hematuria. Respecto a la clasificación clínica se encontró que el $81 \%$ de los pacientes fue cortico sensibles; y 2 pacientes presentaron Enfermedad Renal Crónica (ERC), a 40,3\% se les realizó biopsia renal y una tercera parte presentó infecciones, principalmente de origen respiratorio.

De los 27 pacientes a quienes les realizó biopsia renal, la mayoría $(42,8 \%)$ tuvieron como resultado ECM y en segundo lugar con 32,1\% GEFS. De los pacientes que se les realizó biopsia los que presentan diagnóstico histopatológico de ECM el
Tabla 1. Características Sociodemográficas.

\begin{tabular}{lc}
\hline Datos sociodemográficos & No. (\%) \\
\hline Muestra & 67 \\
\hline Sexo & \\
Masculino & $36(53,7 \%)$ \\
Femenino & $31(46,3 \%)$
\end{tabular}

Edad primera visita (Meses)

Promedio (DE)

$85,94(47,6)$

Edad debut (Meses)

\begin{tabular}{lc}
\hline Promedio $(D E)$ & $46,25(36,02)$ \\
$<12$ Meses & $5(7,5 \%)$ \\
$13-72$ Meses & $51(76,1 \%)$ \\
$73-120$ Meses & $9(13,4 \%)$ \\
$>120$ Meses & $2(3 \%)$
\end{tabular}

Tiempo seguimiento (Meses)

Promedio (DE)

$14,31(9,54)$

Procedencia

Huila

$57(85,1 \%)$

Caquetá

$7(10,4 \%)$

Putumayo

$3(4,5 \%)$

Antecedentes

Personal de ERC

$0(0 \%)$

Familiar de ERC

$5(7,5 \%)$

Neumococo 
R.F.S Revista Facultad de Salud

Enero-Junio de 2015;7(1): 9-16
Caracterización clínica del síndrome nefrótico en infantes de Neival Diana C León, Ana M Agudelo, Jorge Ramos, Milton D Ibarra

Tabla 2. Características Antropométricas.

\begin{tabular}{lcc}
\hline Datos antropométricos & Inicio & Final \\
\hline Talla / Edad & & $56(80,6 \%)$ \\
Normal & $56(83,6 \%)$ & $6(9 \%)$ \\
Riesgo de Talla Baja & $5(7,5 \%)$ & $5(7,5 \%)$ \\
Talla Baja & $6(9 \%)$ & \\
\hline Estado nutricional< 5 años & & $0(0 \%)$ \\
DNT Global severa & $0(0 \%)$ & $1(7,1 \%)$ \\
DNT Global & $0(0 \%)$ & $0(0 \%)$ \\
Riesgo de bajo peso para la edad & $0(0 \%)$ & $7(50 \%)$ \\
Peso Adecuado para la edad & $13(52 \%)$ & $2(14,3 \%)$ \\
Sobrepeso & $6(24 \%)$ & $4(28,6 \%)$ \\
Obesidad & $6(24 \%)$ & \\
\hline IMC > 5 años & & $32(59,25 \%)$ \\
Normal & $22(52,8 \%)$ & $1(1,92 \%)$ \\
Delgadez & $1(2,38 \%)$ & $3(5,8 \%)$ \\
Riesgo para delgadez & $2(4,76 \%)$ & $8(15,4 \%)$ \\
Sobrepeso & $13(30,9 \%)$ & $10(19,23 \%)$ \\
Obesidad & $4(9,52 \%)$ & \\
\hline
\end{tabular}

$100 \%$ continúan siendo cortico-sensibles, no a si los corticoresistentes cuya etiología más frecuente fue la GEFS (66\%). El $3,5 \%$ que corresponde a un paciente presento muestra insuficiente. La mayoría de los pacientes $(95,5 \%)$ recibieron corticoide como tratamiento y en menor proporción ciclosporina $(22,4 \%)$ y ciclofosfamida $(13,4 \%)$. La principal complicación que se presentó en el SN fue la hiperlipidemia seguida por sobrepeso obesidad. El 6\% de los Pacientes presentaron aumento de la tensión arterial durante el debut de la enfermedad y 1 de cada $7(13 \%)$ lo presentó durante el seguimiento. Se obtuvo una tasa de letalidad del $9 \%$, siendo las infecciones la principal causa de muerte.

Se encontró una asociación negativa entre cortico - resistencia y la Tasa de Filtración Glomerular (TFG), lo que significa que si el paciente es cortico resistente disminuye su TFG siendo estadísticamente significativo (Tabla 3).

Como se observa en la Tabla 4 se encontró asociación entre la cortico - resistencia y las complicaciones como el aumento de la tensión arterial al debut o durante el segui- miento, la hipoalbuminemia, hipercolesterolemia, hematuria, biopsia renal y el complemento bajo, todas estas, siendo estadísticamente significativo.

La mayoría de los paciente cortico - resistentes a quienes se les realizo biopsia renal su histopatología fue GEFS $(60 \%)$ y recibieron micofenolato, rituximab y ciclosporina, a diferencia del grupo de los cortico - sensibles.

EL $81 \%$ de los pacientes fueron cortico-sensibles y no se presentó ninguna diferencia estadísticamente significativa entre la edad de debut y su respuesta a los esteroides (Tabla 4).

Existe una menor probabilidad de supervivencia en el grupo de Cortico - resistente, comparado con el grupo de sensibilidad al corticoide. Según la gráfica de supervivencia de Kaplan Meier, la sobrevivencia en los Pacientes con SN a los 8 meses de seguimiento es mayor en los cortico-sensibles que en los cortico-resistentes ( $90 \%$ Vs $60 \%$ ).

Los pacientes fallecidos tuvieron en promedio menos recaídas comparado con los vivos, existiendo una diferencia estadísticamente significativa $(0,04)$ (Tabla 5).

Tabla 3. Correlación de TFG en Cortico - sensible y Cortico - resistente.

\begin{tabular}{lccc}
\hline & Corticosensible & Corticoresistente & Correlación Spearman (P) \\
\hline $\begin{array}{l}\text { TFG AL INICIO } \\
\text { Media (DE) }\end{array}$ & $148,91(48,49)$ & $146,08(52,58)$ & $-0,05(>0,05)$ \\
\hline $\begin{array}{l}\text { TFG AL AÑO } \\
\text { Media (DE) }\end{array}$ & $147,33(49,38)$ & $104(21,6)$ & $-0,35(0,03)$ \\
\hline
\end{tabular}


Tabla 4. Factores Asociados a Resistencia o Sensibilidad del Corticoide.

\begin{tabular}{|c|c|c|c|c|}
\hline Datos clinicos & Corticosensible & Corticoresistente & $X^{2}(P)$ & R.P (IC 95\%) \\
\hline Muestra & 54 & 13 & & \\
\hline Edad debut & $47,6(35,9)$ & $16,67(27,15)$ & & $N / A$ \\
\hline Media (Desviación Estándar) & & & $-0,22(0,07)^{*}$ & \\
\hline No. RECAIDAS & $3,69(3,8)$ & $3,33(5,7)$ & & $N / A$ \\
\hline Media (Desviación Estándar) & & & $-0,09(0,45)^{*}$ & \\
\hline Factores asociados & & & & N/A \\
\hline Antecedente Familiar & $3(5,6 \%)$ & $2(15,4 \%)$ & $0,38(0,53)$ & \\
\hline Masculino & $30(55,6 \%)$ & $6(46,2 \%)$ & $0,09(0,55)$ & \\
\hline Infección Respiratoria & $18(33,3 \%)$ & $3(23,1 \%)$ & $0,14(0,74)$ & \\
\hline Infección en Piel & $0(0 \%)$ & $1(7,7 \%)$ & $0.60(0,43)$ & \\
\hline Complicaciones & & & & $\mathrm{N} / \mathrm{A}$ \\
\hline HTA al debut & $2(28,6 \%)$ & $2(18,2 \%)$ & $0,26(1)$ & \\
\hline HTA seguimiento (Debut) & $2(33,3 \%)$ & $2(16,7 \%)$ & $1,32(0,59)$ & \\
\hline Hiperlipidemia & $20(37 \%)$ & $5(38,5 \%)$ & $0,01(1)$ & \\
\hline Obesidad & $20(37 \%)$ & $2(15,4 \%)$ & $1,35(0,19)$ & \\
\hline \multicolumn{5}{|l|}{ Paraclinicos } \\
\hline Hipoalbuminemia & $10(18,5 \%)$ & $7(53,8 \%)$ & $5,16(0,01)$ & $3,43(1,3-8,7)$ \\
\hline Hipercolesterolemia & $10(18,5 \%)$ & $7(53,8 \%)$ & $5,16(0,01)$ & $3,43(1,3-8,7)$ \\
\hline Hematuria & $3(5,6 \%)$ & $5(38,5 \%)$ & $7,88(<0,01)$ & $4,6(1,9-10.6)$ \\
\hline Biopsia Renal & $17(31,5 \%)$ & $10(76,9 \%)$ & $7,23(<0,01)$ & $4,9(1,4-16,3)$ \\
\hline Complemento Bajo & $0(0 \%)$ & $3(30 \%)$ & $8,8(<0,01)$ & $6,4(3,6-11,3)$ \\
\hline
\end{tabular}

* Correlación Spearman.

Tabla 5. Factores Asociadas a la Mortalidad en el S.N.

\begin{tabular}{|c|c|c|c|c|}
\hline Datos clinicos & Fallecido & Vivo & $X^{2}(P)$ & R.P (IC 95\%) \\
\hline Muestra & 6 & 61 & & \\
\hline Factores asociados & & & & N/A \\
\hline Corticoresistente & $4(66,7 \%)$ & $9(14,8 \%)$ & $6,38(0,01)$ & $8,3(1,7-40,5)$ \\
\hline Masculino & $3(50 \%)$ & $33(54,1 \%)$ & $0,03(1)$ & $0,8(0,18-3,9)$ \\
\hline Infección Respiratoria & $3(50 \%)$ & $18(29,5 \%)$ & $1,06(0,30)$ & $2,19(0,4-9,9)$ \\
\hline Infección en Piel & $0(0 \%)$ & $1(1,6 \%)$ & $0.1(0,75)$ & N/A \\
\hline \multicolumn{5}{|l|}{ Complicaciones } \\
\hline HTA al debut & $3(50 \%)$ & $1(1,6 \%)$ & $22,8(<0,01)$ & $15,75(4,5-54,4)$ \\
\hline HTA seguimiento & $2(33,3 \%)$ & $2(3,3 \%)$ & $11,52(0,02)$ & $7,8(2,01-30,8)$ \\
\hline Hiperlipidemia & $3(50 \%)$ & $22(36,1 \%)$ & $0,05(0,66)$ & $1,68(0,36-7,6)$ \\
\hline Obesidad & $1(16,7 \%)$ & $21(34,4 \%)$ & $0,18(0,65)$ & $0,40(0,05-3,2)$ \\
\hline
\end{tabular}


R.F.S Revista Facultad de Salud

Enero-Junio de 2015;7(1): 9-16
Existe asociación entre los cortico - resistentes e HTA con la mortalidad de los niños y niñas de SN No se encontró ninguna diferencia entre dislipidemia y obesidad con el fallecimiento.

Un factor importante en la letalidad de los pacientes con SN es la Tasa de Filtración Glomerular al inicio de atención; la mitad de los pacientes que fallecieron tenían una TFG de 125 y 128 en los sensibles y resistentes respectivamente; mientras los vivos tuvieron una mediana de 138 en resistentes y 140 en los sensibles (Figura 2).

\section{Discusion}

Teniendo en cuenta la población menor de 15 años proyectada para el Departamento del Huila en el $2012^{(3)}$, se tuvo una prevalencia de 19,7 x 100,000 niños, por encima a lo reportado en la literatura mundial de 2 por $100,000^{(2)}$. Llama la atención esta prevalencia elevada, teniendo en cuenta que podría haber un sub-registro en nuestro estudio, correspondiente a los pacientes que no son atendidos en la ciudad de Neiva, lo cual implica que esta patología es más frecuente en nuestra población.

La edad de presentación fue similar a la reportada en la literatura, en la que el grupo más frecuente son los pacientes menores de 5 años; los pacientes debutaron en un promedio de edad a los 46,2 meses y su mayor proporción fue en niños de 1 6 años con el $76,1 \%{ }^{(4)}$. La distribución por sexo no concuerda con los datos internacionales (2:1) en los que se observa predominio de hombres; en nuestro estudio la relación hombre-mujer fue de 1,16:1 ${ }^{(4),(5)}$. El tiempo de seguimiento en promedio fue de 14 meses, menor al reportado por los estudios de Medellín y Taiwán (5,4 -7,7 años respectivamente).

Como factor determinante negativo se encontró que el $28,4 \%$ de los pacientes no tuvieron vacuna antineumocóccica; medida profiláctica que recomiendan las guías de atención basadas en la evidencia ${ }^{(6),(7)}$, teniendo en cuenta que estos pacientes presentan alteraciones de tipo inmunológico que incluyen la deficiencia de ozonización de gérmenes encapsulados como Streptococcus pneumoniae.

La población del Huila menor de 5 años con talla baja para la edad en el año 2009 fue 11,5\% y la desnutrición (DNT) global es del 6,30\% según estadísticas de la Secretaria de Salud Departamental del Huila ${ }^{(8)}$. Para el presente estudio se encontró una proporción de niños de baja talla para la edad del $9 \%$ al inicio y $7,5 \%$ al final del seguimiento, y de DNT global de $7,10 \%$, mucho menor comparado con el estudio realizado en Matanzas que reporta una tasa de retardo ponderal del $14 \%{ }^{(4)}$.

Respecto al estado nutricional de los niños mayores de 5 años, no se evidenció una diferencia significativa al inicio y final del seguimiento; el único cambio que llama la atención fue la reducción de la proporción de sobrepeso y el aumento de los obesos al final del seguimiento, con un porcentaje de obesidad del $34 \%$, mayor al reportado previamente ${ }^{(9)}$ del año 1993, en el que se evidenció un porcentaje de obesidad del $22 \%$. Esto apoya la evidencia de que la terapia con corticoides a largo plazo genera problemas de obesidad.

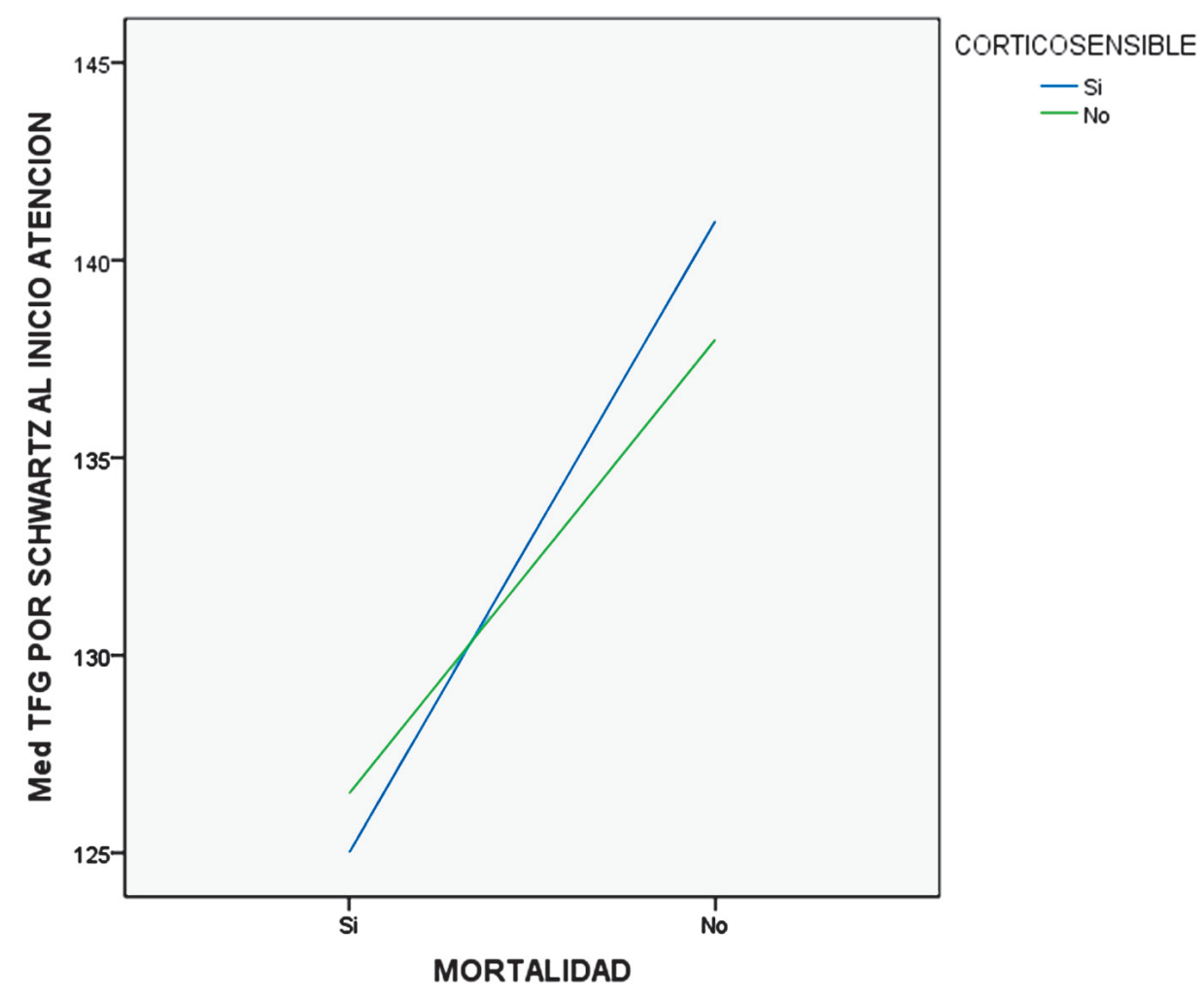

Figura 2. Mortalidad según T.F.G en Cortico - Sensible y Cortico - Resistente. 
La ganancia promedio de peso y talla en un periodo de seguimiento de 14 meses corresponde a lo esperado en la población pediátrica, según los datos suministrados por UNICEF ${ }^{(10)}$.

Los pacientes que debutaron con SN todos presentaron los cuatro principales criterios diagnósticos comentados en la literatura, que son, edema, hipoalbuminemia, proteinuria e hipercolesterolemia. Algunos de los hallazgos paraclínicos que sugieren formas histopatológicas diferente a ECM como son: hematuria, C3 bajo, perfil inmunológico positivo, entre otros, fueron indicadores que estuvieron presentes en una pequeña proporción de los pacientes $(10 \%)$. El perfil infeccioso fue negativo para todos.

Preocupa la tasa de letalidad elevada ( $9 \%$ ) comparada con la epidemiología nacional según lo reportado en el estudio del valle $(1,6 \%)$ y del $5,7 \%$ en el estudio realizado en Medellín. La principal causa de mortalidad fueron las infecciones, lo cual difiere de lo encontrado en la literatura nacional, en donde la principal causa de mortalidad fue la enfermedad renal crónica seguida por las causas infecciosas, como lo reporta el estudio de Medellín ${ }^{(11)}$. Por lo anterior, se deberá garantizar el estricto cumplimiento y la adhesión al aislamiento protector a nivel hospitalario en estos pacientes, debido al estado de inmunosupresión en que se encuentran por su enfermedad y su tratamiento.

En cuanto a las principales complicaciones encontradas en este estudio, se observó una mayor frecuencia de presentación de dislipidemia (37\%), seguida por la obesidad (35\%), las infecciones respiratorias (31\%), e HTA (13\%). Al compararlo con otras series se encuentran diferencias como en el estudio de Medellín donde la principal complicación fue infecciosa seguida de HTA ${ }^{(11)}$; en el estudio Taiwanés, reportan como principal causa las infecciosas (IVU), seguido de injuria renal aguda y menos frecuente complicaciones trombóticas y encefalopatía hipertensiva.

La hipertensión arterial como complicación se presentó con una frecuencia de hasta el $13,5 \%$ en nuestros pacientes con edad de 4 años; esto resulta ser tres veces más que lo esperado para la población normal de esta edad, según lo refiere la literatura ${ }^{(12)}$.

La progresión a enfermedad renal crónica se encontró solamente en dos pacientes (3\%), quienes debutaron a los 4 y 8 años de edad, con tiempo de evolución de enfermedad de 8 y 4 años y reporte histopatológico de GEFS y nefropatía membranosa respectivamente. Hallazgos similares de frecuencia de ERC reporta la literatura, como en el estudio del Valle $(2,4 \%)$, Matanzas $(2 \%)$, Taiwán $(3,6 \%)$, pero difiere del estudio de Medellín (9\%); esto puede explicarse por el mayor tiempo de seguimiento en este último estudio.

La frecuencia encontrada de cortico-resistencia del 19\% es similar a la descrita por otras revisiones nacionales que van del 11 al $20 \%{ }^{(13)(11)}$. Se encontró que por cada niño resistente hay cuatro cortico-sensibles. La mayoría de los pacientes con síndrome nefrótico responden a los corticoides, similar a lo encontrado en nuestro estudio.

Dentro de los paraclínicos se encontró que los pacientes cortico-resistentes tienen más de tres veces oportunidades de tener hipoalbuminemia, hipercolesterolemia, hematuria, y complemento bajo comparado con los cortico-sensibles. Esto se asocia a la mayor posibilidad de recaídas y de tener formas histológicas diferentes a la enfermedad de cambios mínimos en los pacientes cortico-resistentes. La literatura reporta que hay una mayor frecuencia de hematuria de acuerdo al hallazgo histopatológico, que presenta un $22,7 \%$ de cambios mínimos, 48,4\% en GEFS y 58,5\% en otras formas como en la membrana-proliferativa.

El tratamiento de primera línea fueron los corticoides en todos los pacientes, excepto en los dos pacientes con síndrome nefrótico congénito, en quienes no está indicado este medicamento ya que su etiología está dada por anomalías en los genes codificadores de proteínas específicas del podocito (con excepción de los secundarios a enfermedades infecciosas), que origina daño estructural y pérdida de la barrera de filtración glomerular, y no responden al tratamiento con corticoide u otro agente citotóxico.

El 25\% (17/67) de los niños requirieron tratamiento inmunosupresor por cortico-resistencia, cortico-dependencia y/o recaídas frecuentes; en un $88 \%$ de los casos (15/17) el inmunosupresor elegido fue la ciclosporina, y en el $52 \%$ de los casos recibieron ciclofosfamida. Otras opciones de tratamiento fueron micofenolato y rituximab en pacientes que no respondieron a ciclosporina. El uso de ciclosporina ha aumentado en los últimos tiempos debido a una mayor evidencia de respuesta en los pacientes con glomeruloesclerosis focal y segmentaria, según la guía del 2012 de KDIGO.

El tratamiento del SN primario pretende lograr y mantener una remisión de la enfermedad, balanceando los riesgos y beneficios de las drogas eficaces en su manejo. Si no se logra una remisión adecuada, existe el riesgo de complicaciones graves, como infecciones bacterianas, eventos tromboembólicos y desnutrición proteico-calórica, entre otras (6). El pronóstico a largo plazo está condicionado en gran medida por la respuesta a corticoides. Los pacientes sensibles a ellos evolucionan habitualmente hacia la resolución de su enfermedad con preservación de la función renal. A pesar de que más del $90 \%$ de los pacientes responde a prednisolona, un alto porcentaje (50-70\%) de ellos cursa con recaídas frecuentes (SNRF) o se transforma en cortico-dependiente (SNCD). Los pacientes con resistencia a esteroides en su mayoría corresponden a GEFS, entre los cuales alrededor del $50 \%$ desarrollará insuficiencia renal crónica (12).

La letalidad en este grupo de pacientes se asoció con la resistencia al corticoide, con HTA en el debut y seguimiento, y llama la atención que los pacientes fallecidos presentaron menores recaídas comparado a los vivos; esto se podría deber a que los primeros ocho meses es una fase aguda para la mortalidad en este grupo de pacientes, lo que ocasiona que no presenten mayor número de recaídas.

Nuestro estudio tuvo algunas limitaciones como la evaluación retrospectiva que no permite hacer controles adecuados; sin embargo, a nivel local, es el primer estudio realizado en la población infantil atendida por el servicio de nefrología pediátrica en nuestra región, con un periodo de seguimiento de casi tres años, lo que aporta información valiosa sobre el comportamiento local de la enfermedad. 
R.F.S Revista Facultad de Salud

Enero-Junio de 2015;7(1): 9-16
Se encontraron datos similares a los informados en la literatura mundial y local en cuanto a la distribución etaria y edad de debut, con una buena respuesta en general al tratamiento esteroide. Sin embargo, en cuanto a los demás inmunosupresores usados se requieren nuevos estudios para determinar cuál es el medicamento más seguro y efectivo para el tratamiento de los niños con SN.

\section{Agradecimientos}

Los autores expresan sus agradecimientos al Hospital Universitario Hernando Moncaleano Perdomo por su colaboración para realizar satisfactoriamente esta investigación y producción de conocimiento.

\section{Referencias}

1. Nephrotic syndrome in children. . Bagga A, Mantan M. s.I. Indian J Med Res. 2005, Vol. 122.

2. Current conceptsofthepodocyteinnephroticsyndrome. Wen Y.Ding, MoinA.Saleem. Korea : Kidney Res Clin Pract, 2012;31:87-93.

3. Departamento Administrativo Nacional de Estadística DANE. Proyección Población por Departamentos de Colombia. Censo 2005. Bogotá: s.n., 2005.

4. Síndrome Nefrótico un studio de 18 años. Montell O., Vidal A., Sánchez C., Méndez A. 5, Matazas: Revista Médica Electrónica, 2009, Vol. 31.

5. Childhood nephrotic syndrome: change in pattern and response to steroids. Anochie I, Eke F, Okpere A. 12, s.l. J Natl Med Assoc, 2006;98:1977-1981.
6. Nephrotic Syndrome. Bagga, Aditi Sinha \& Arvind. 8, India. Indian J Pediatr, 2012, Vol. 79.

7. Management of Childhood Onset Nephrotic Syndrome. Debbie S. Gipson, Susan F. 2, s.I. PEDIATRICS. 2009, Vol. 124.

8. Secretaría de Salud Departamental del Huila. Situación de Salud en el Huila. Neiva: s.n., 2009.

9. Long-term ciclosporin treatment in children with steroiddependent nephrotic syndrome . Tanaka, Ryojiro. Korea: Pediatrics Nephrology, 1993.

10. Vigilancia del Crecimiento en niños y niñas. UNICEF.

11. Evolución a largo plazo de los niños con diagnóstico de síndrome nefrótico atendidos en el Hospital Universitario San Vicente de Paúl, Medellín, entre enero de 1960 y diciembre de 2009. Atehortúa, Pahola Shyrley y Vilma, Piedrahíta. 2, Medellin: latreia, 2013;26:127-135.

12. Pediatric ambulatory blood pressure monitoring: diagnosis of hypertension. Chaudhuri., Abanti. s.I. Pediatr Nephrol , 2013, Vol. 28, págs. 995-999.

13. Síndrome nefrótico: experiencia en el servicio de nefrología pediátrica de la Fundación Cardioinfantil y Clínica Infantil Colsubsidio. Gastelbondo R, Benavides J, Botero D. Bogotá. Actualizaciones Pediátricas, 2003, Vol. 13.

14. Changing incidence of glomerular diseases in adults. Braden GL, Mulhern JG, O'Shea MH, Nash SV, Ucci AA, Jr., Germain MJ. 5, s.I. Am J Kidney Dis, 2000, Vol. 35. 